\title{
Planned Observations of Hot Gas in the LISM
}

\author{
R. McLean, J.C. Green, and K.S. Gunderson \\ Center for Astrophysics and Space Astronomy, University of Colorado, Boulder, \\ U.S.A.
}

\begin{abstract}
We plan to observe hot gas in the local interstellar medium (LISM) at high resolution using a rocket-borne spectrograph that can simultaneously observe OVI, NV and CIV at resolutions of $1-2 \mathrm{~km} \mathrm{~s}^{-1}$. Data from a single flight will have sufficient signal to noise ratio to detect a broad $50 \mathrm{~km} \mathrm{~s}^{-1}$ component down to a column density of $N \geq 0.5 \times 10^{13} \mathrm{~cm}^{-2}$. High-velocity components as fast as $300 \mathrm{~km} \mathrm{~s}^{-1}$ can be detected. New high resolution observations of hot gas absorption line profiles will probe the kinematics of the local bubble, and comparison between the carbon, nitrogen and oxygen profiles will allow discrimination between the various production models.
\end{abstract}

\section{Background}

High stage ions trace hot gas in the interstellar medium. Measurements of OVI absorption are primarily from observations by Copernicus along 72 sight lines (Jenkins (1978)). The original analysis of these data found that OVI was clumped in columns of $10^{13} \mathrm{~cm}^{-2}$ with an average separation of $165 \mathrm{pc}$. However, this analysis did not allow for a common component along all sight lines. Adding a local component of $1.6 \times 10^{13} \mathrm{~cm}^{-2}$ with an rms velocity of $12 \mathrm{~km} \mathrm{~s}^{-1}$ improves the fit to the data (Shelton and Cox (1994)). Detailed profile analysis of this component is not possible with the $14 \mathrm{~km} \mathrm{~s}^{-1}$ resolution of Copernicus.

By adding in data from other high stage ions, it is possible to constrain the production mechanism for the hot gas. Production via radiative cooling, conductive heating and turbulent mixing layers can be differentiated by comparing the ratio of the column densities of pairs of ions (Shelton (1996)). Also possible is a mechanism where high stage ions are frozen in at cooler temperatures (Breitschwerdt and Schmutzler (1994)). Higher resolution data that make a direct comparison between OVI, NV and CIV profiles can further constrain models.

\section{Objectives}

We want to look at the kinematics of the hot gas in the local bubble by making simultaneous observations of OVI, NV and CIV in absorption at resolutions of $1-2 \mathrm{~km} \mathrm{~s}^{-1}$ with a signal to noise ratio of 25 . In addition to 

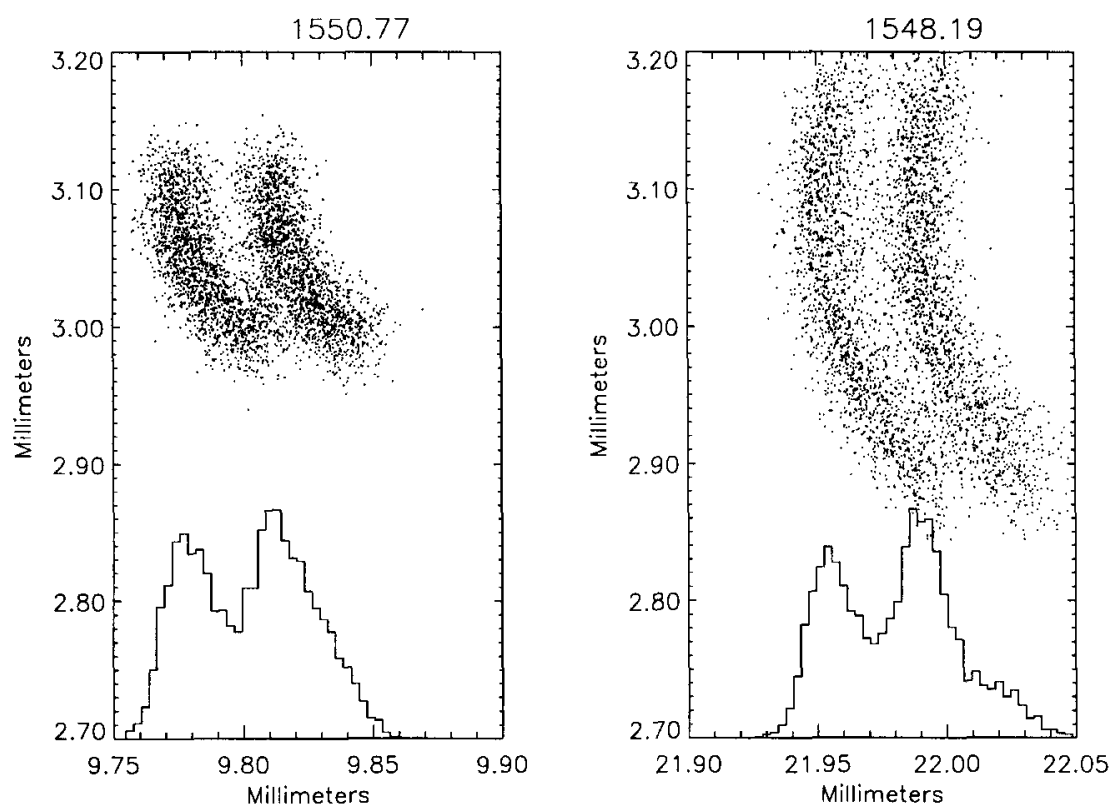

Fig. 1. Raytraces of the C IV doublet as seen by the HotCONES instrument. The two spots at each wavelength are separated by a resolution of 200,000 . The histograms show line profiles in the $\mathrm{x}$ dimension only.

profile fitting, we will be able to detect a broad $50 \mathrm{~km} \mathrm{~s}^{-1}$ component with $3 \sigma$ uncertainty down to a limiting column density of $0.5 \times 10^{13} \mathrm{~cm}^{-2}$ as well as detect components as fast as $300 \mathrm{~km} \mathrm{~s}^{-1}$.

\section{Instrument}

The Hot Carbon, Oxygen, Nitrogen Echelle Spectrograph (HotCONES) uses a low order echelle as an objective grating with a cross-dispersing and focusing parabolic secondary to take simultaneous spectra at the widely separated transitions of CIV, OVI and NV. The low order echelle is ruled at a line density of 2,880 lines per millimeter with a blaze angle 63 degrees. Ruled in this way, fourth, fifth, and sixth orders have the high dispersion of an echelle without having to work with many overlapping orders. This concentrates the limited bandwidth of the detector around the high stage ion absorption lines without covering all of the intermediate wavelengths.

Raytraces of each of the transitions are shown at a separation of $\lambda / \delta \lambda$ of 200,000 . The spectrograph will use a 25 micron resolution microchannel plate detector. The blur of the detector has been added to the raytraces shown. 

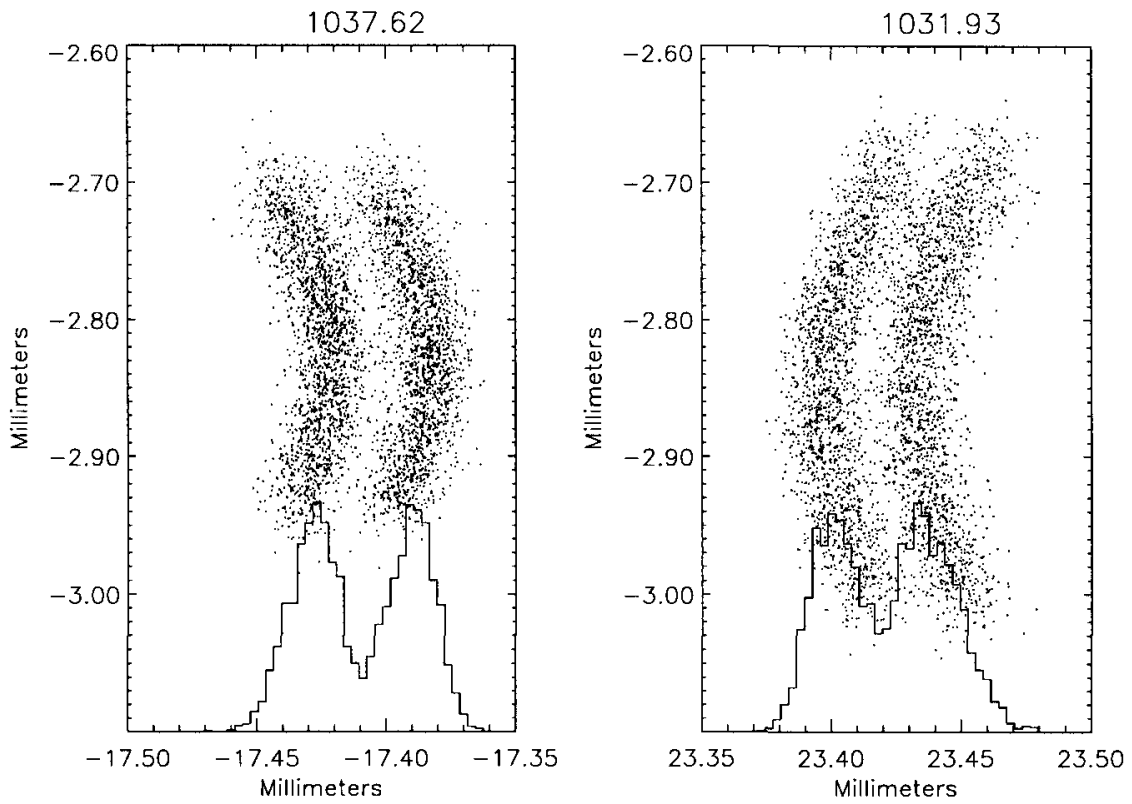

Fig. 2. Raytraces of the O VI doublet as seen by the HotCONES instrument. The two spots at each wavelength are separated by a resolution of 200,000 . The histograms show line profiles in the $\mathrm{x}$ dimension only.

As a conservative estimate, the HotCONES experiment should have an effective area of at least $1.5 \mathrm{~cm}^{2}$. A sketch of the spectrograph packaged into a 22 inch diameter sounding rocket is shown in Fig. 4. In the roughly 300 seconds available from this sub-orbital platform, HotCONES can only observe bright stars to get a signal to noise ratio of 25 . In the first two flights we plan to observe $\alpha$ Vir and $\delta$ Ori.

\section{References}

Breitschwerdt D., \& Schmutzler T. (1994): Nature 371, 774

Jenkins E.B. (1978): ApJ 219, 845

Shelton R.L., \& Cox D.P. (1994): ApJ 434, 599

Spitzer, L. (1996) ApJ 458, L29 

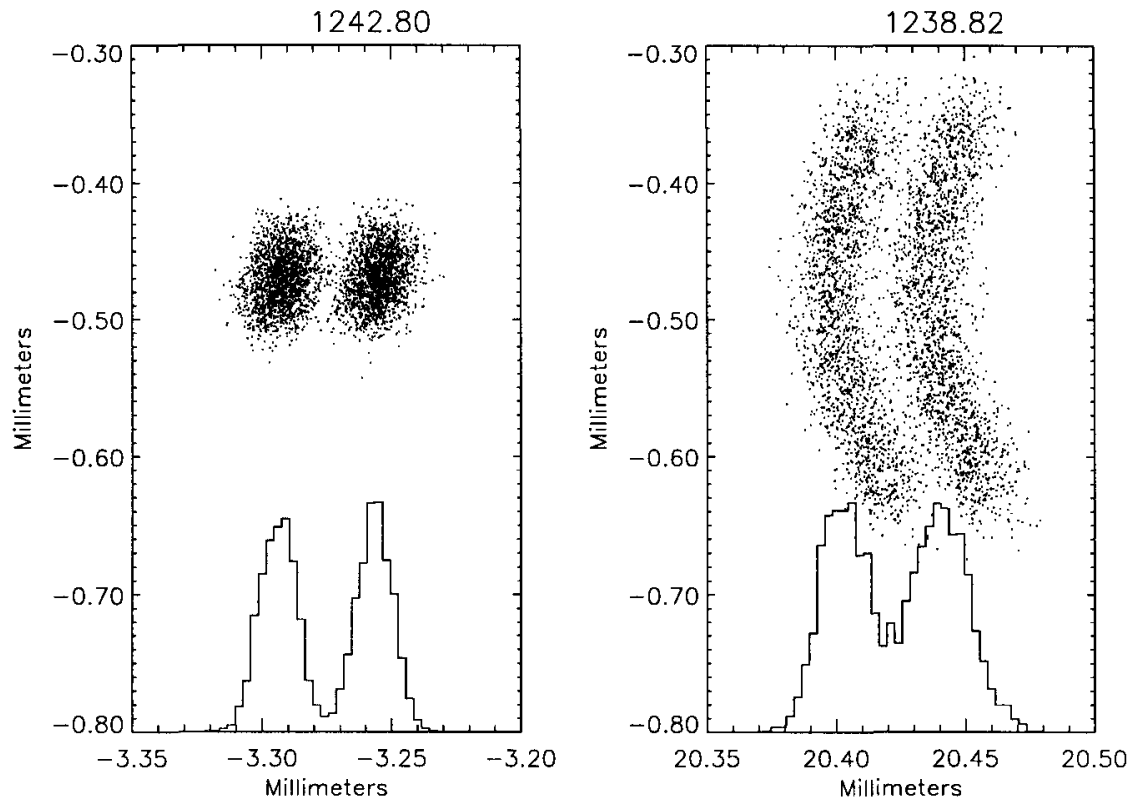

Fig. 3. Raytraces of the N V doublet as seen by the HotCONES instrument. The two spots at each wavelength are separated by a resolution of 200,000 . The histograms show line profiles in the $\mathrm{x}$ dimension only.

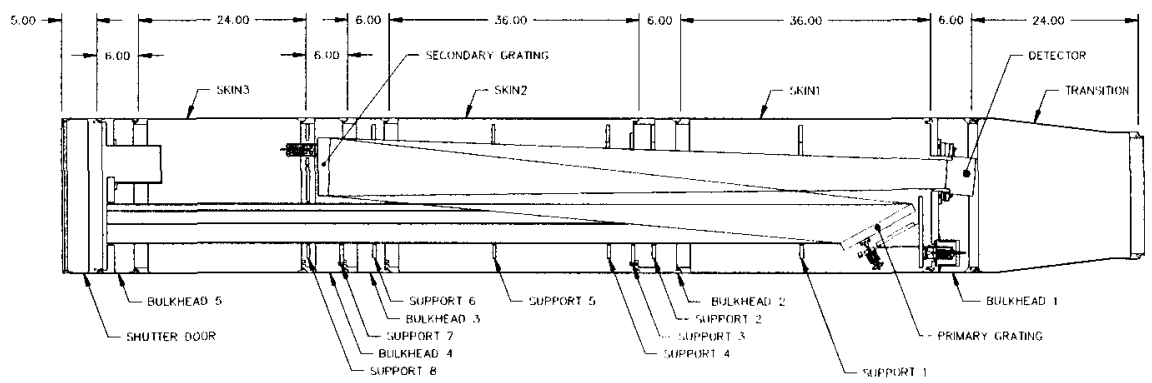

Fig. 4. Section view of the HotCONES instrument. 